\title{
Pharmacists' attitudes towards a pharmaceutical assessment screening tool to help prioritise pharmaceutical care in a UK hospital
}

\author{
Katherine J E Saxby, ${ }^{1}$ Ruth Murdoch, ${ }^{1}$ John McGuinness, ${ }^{1}$ Douglas T Steinke, ${ }^{1,2}$ \\ Steven D Williams ${ }^{1,2}$
}

'University Hospital of South Manchester, Manchester, UK ${ }^{2}$ Manchester Pharmacy School, University of Manchester, Manchester, UK

Correspondence to Steven D Williams, Pharmacy Department, University Hospital of South Manchester, Manchester M23 9LT, UK; steven.williams@dorset.nhs.uk

Received 7 August 2016 Revised 23 November 2016 Accepted 28 November 2016 Published Online First 20 December 2016

\section{EAHP Statement 4: Clinical} Pharmacy Services
ABSTRACT

Objective To establish the thoughts of pharmacists using the pharmaceutical assessment screening tool (PAST) when assigning a patient acuity level (PAL) and establish other decision factors. A PAL is a pharmaceutical assessment of a patient (lowest $=1$ to highest=3), higher PALs highlight the requirement for a more intensive pharmaceutical input to reduce potential harm.

Method A questionnaire designed to elicit attitudes about the PAST was circulated to 32 pharmacists working in a 900 bed UK university teaching hospital. Respondents were asked to document what PAL they would assign for six theoretical patient cases with an explanation. The data collected was analysed using Microsoft Excel and further analysis was undertaken about the strength of agreement to PAST using the $\kappa$ statistic using Stata V.12 (StataCorp, Texas, USA).

Results The questionnaire was completed by $28 / 32$ pharmacists ( $87.5 \%$ response rate). The mean confidence (SD) for assigning a PAL was $81 \%( \pm 20 \%)$. $26 / 28$ pharmacists $(93 \%)$ agreed or strongly agreed that professional judgement guided them most when allocating a PAL. The PAL assigned to the case studies presented both overestimations and underestimations compared with the guidance but overall the strength of agreement was considered to be 'fair' ( $\kappa=0.202$ ).

Conclusions Pharmacists feel confident about using PAST to help them assign a PAL. However, the use of professional judgement to assign an acuity level overrides any predicted level from PAST.

\section{INTRODUCTION}

Assessment tools to help guide the levels of care and staffing required on hospital wards are in regular use for medical and nursing staff in UK hospitals. $^{1}$ Tools to help predict patient requirements for pharmaceutical care in hospitals are however poorly developed. A small number of pharmaceutical assessment screening tools (PASTs) have been designed and introduced to prevent adverse drug events in response to inadequate pharmacy services causing critical medication safety incidents ${ }^{3}$ to provide clinical pharmacy review in a more timely and targeted manner ${ }^{4} 5$ and identify complex patients in need of referral to a more experienced clinical pharmacist. ${ }^{6}$

Our hospital pharmacy department has designed its own PAST in a bid to help teams of clinical pharmacists prioritise both the frequency and the seniority of pharmacists performing patient reviews. ${ }^{7}$ The development of the PAST as a screening tool is described elsewhere. ${ }^{7}$ Briefly, patient acuity levels (PALs) (lowest $=1$ to highest $=3$ ) are calculated by the ward pharmacist manually using the tool (see figure 1) and patients with higher PALs are expected to receive more intensive pharmaceutical input to reduce the risk of adverse drug events. However, it was only partially successful as the documented PAL only matched the expected acuity level derived from using the tool in $57 \%$ of patients. ${ }^{7}$ The clinical services managers felt that if the department was going to reliably use the assessment tool to prioritise pharmaceutical care there was a need to refine the tool by finding out why pharmacists did not appear to follow the guidance in all patients.

The aim of this study was establish what pharmacists knew and thought about the current PAST to assess the PAL and what factors they used to assign a level on a daily basis.

\section{METHOD}

All 32 pharmacists who provided ward-based pharmaceutical care for medical, surgical maternity and paediatric patients in the 900 bed UK university teaching hospital were invited to take part in the study. Pharmacists working solely on intensive care units (ICUs) and in the cystic fibrosis centre (CF) were excluded as all patients in ICUs and CF are automatically assigned the highest PAL. Pharmacists covering mental health or community step-down units were also excluded, as they do not currently use the tool in practice.

A questionnaire was designed to elicit the attitudes and opinions of the pharmacists towards PAST using questions featuring a five-point Likert scale and an opportunity for free text response. Questions aimed to understand the pharmacists confidence and perceived usefulness of PAST and whether improvements could be made. Six theoretical case studies of patients were devised by the clinical research team based on actual patients seen at the hospital (see figure 2). The case studies consisted of two level 3 (L3) patients, three level 2 (L2) patients and one level 1 (L1) patient. Pharmacists were asked to document what PAL they would assign each patient, if they saw them on their ward, and to give an explanation of why that particular acuity level was chosen.

The initial questionnaire was pretested with five pharmacists; minor changes were then made to the wording of a number of questions. After the amendments, the questionnaire was distributed to all
To cite: Saxby KJE,
Murdoch R, McGuinness J, et al. Eur J Hosp Pharm 2017;:24:315-319. 
Figure 1 Pharmaceutical assessment screening tool. Anti-TNF, anti-tumor necrosis factor; IBD, inflammatory bowel disease; NOAC, new oral anticoagulant.

\section{Guide for initial allocation of Pharmaceutical Assessment Level}

Level 3 Patients

- Patient is severely unwell with $>1$ acutely decompensated organ (kidney, liver, heart, lung, bone marrow, brain)

- Patient is on a High Risk Medicine (HRM) or a medicine requiring Therapeutic Drug Monitoring (TDM) plus 1 acutely decompensated organ

- Patients stepped down from intensive care unit or under review by ICU outreach team

- An acute admission managed (or jointly managed) by an Infectious Diseases (ID) Consultant

- Cystic Fibrosis, Organ transplant or HIV patients

Being considered for Home IVs / ASPIRE service

PD patient on Apomorphine pump

- On a high cost PbR medicine (eg Posconazole) or being considered for one (e.g. Anti TNF for IBD)

- Patient's condition or drug therapy regimen outside of the competency of the band 6 pharmacist

\section{Level 2 Patients}

- Patients is on a (HRM)*

Patient is on a medicine requiring (TDM)

- Patient has 1 acutely decompensated organ

Level 1 Patients

- Low risk medicines only
- Limited co-morbidities
- Medicine regimen stable
- Clinically stable
Medically fit and awaiting care packages for discharge

*High Risk Medicines (HRM)

- Anti-Coagulants Warfarin and NOACs (excluding enoxaparin $\leq 40 \mathrm{mg}$ daily)

- Insulin

- Regular strong Opiates (excluding Codeine/ Dihydrocodeine, Tramadol)

- Drugs with a Narrow Therapeutic Index

- Chemotherapy

- Antiretrovirals

- Clozapine pharmacists both by email and by internal post. Participants were given 1 week to anonymously complete and return the questionnaire to the lead researcher (KJES). Three email reminders were sent during the week to try to improve the response rate.

The data collected from the questionnaires was analysed using Microsoft Excel. The level of agreement between all respondents and the expected PALs was assessed using the $\kappa$ statistic using Stata V.12 (StataCorp, Texas, USA).

As this was a service evaluation project, ethics approval was deemed unnecessary by research and development department of the hospital.

\section{RESULTS}

\section{Pharmacist demographics}

Within the pharmacy department, 28/32 pharmacists completed the questionnaire giving a response rate of $87.5 \%$. The respondents comprise 5/28 (18\%) pharmacists qualified for $<1$ year, 7/28 (25\%) pharmacists qualified for $1-4$ years, 6/28 (21\%) pharmacists qualified for $>4-10$ years and 10/28 (36\%) pharmacists qualified for $>10$ years.

The results from the questions are presented in table 1.

Comments from the open text section of the questionnaire included:

- What is the main factor when deciding on the PALs of a patient?

It should be noted that I don't get regular technician support so although a patient doesn't always require a pharmacist review, they need to be seen by someone for new medication, I level patients higher than expected sometimes to ensure they are seen. (Pharmacist qualified for $>4-10$ years)

- How do you think the PALs could be improved?

To have a separate pharmacy handover sheet with jobs to follow up for each patient.

(Pharmacist qualified for $<1$ year) 
Case study 1:

Female
DOB: 06/08/1942
Allergies: NKDA
PMH: epilepsy, AF, Alcohol related problems, depression
PC: Seizure
Bloods: eGFR- $53 \mathrm{ml} / \mathrm{min} / 1.73$ sq. $\mathrm{m}, \mathrm{Hb} 108 \mathrm{~g} / \mathrm{l}$, Plt- $193 \times 10^{9} / \mathrm{l}, \mathrm{INR} 4.8$
Medication at home:
- Isosorbide mononitrate MR $30 \mathrm{mg}$ once daily
- Levetiracetam $500 \mathrm{mg}$ twice daily
- Thiamine $50 \mathrm{mg}$ four times a day $\rightarrow$ hold whilst administering IV
thiamine
- Venlafaxine $75 \mathrm{mg}$ once daily
Started in hospital:
- Lorazepam $1 \mathrm{mg}$ when required maximum $2 \mathrm{mg}$ daily
- Pabrinex intravenous high potency solution for injection $5 \mathrm{ml}$ in $50 \mathrm{ml}$
physiological saline (thiamine $250 \mathrm{mg}$, riboflavin $4 \mathrm{mg}$, pyridoxine $50 \mathrm{mg}$,
ascorbic acid $500 \mathrm{mg}$, nicotinamide $160 \mathrm{mg}$, anhydrous glucose
1000mg)
Vitamin B co-strong two tablets twice daily

Which pharmaceutical assessment level would this patient be?

L1

L2

Figure 2 Example case study. eGFR, estimated glomerular filtration rate; INR, International normalized ration.

The biggest problem is updating the PAL status. It is easy to allocate a PAL on admission to prioritise attention but if it is not updated during admission patients can get overlooked.

(Pharmacist qualified for $>4-10$ years)

It is most difficult when covering wards for one day as sometimes I am unsure of how up to date the PAL is for each patient.

(Pharmacist qualified for $>4-10$ years)

Table 2 highlights the differences between respondents' reporting PAL and the level expected using the PAST.

The overall strength of agreement between respondents and the expected acuity levels was considered to be 'fair' $(\kappa=0.202){ }^{8}$

As it was thought that the experience and knowledge of a pharmacist could affect the allocation of a PAL, the strength of agreement when allocating PAL extremes between pharmacist groups with different levels of experience was also tested. The combination of L1 and L3 was used as one extreme and compared with L2. Results presented in table 3.

\section{DISCUSSION}

Overall pharmacists were very confident about using PAST, and agreed that it helped to assign an acuity level and monitor the pharmaceutical needs of inpatients. However, pharmacists appear to rely more on professional judgement than the tool itself to assign a PAL, regardless of experience. The differences in PALs assigned to the case study patients gave a good illustration of the variations between pharmacists and likely use of professional judgement.

In four of the case studies, 10/28 (36\%) of pharmacists allocated a higher level than recommended in the guidance tool and
Table 1 Questionnaire responses

Question Results

What is the main factor when deciding $2 / 28(7 \%)$ used the PAL guidance as on the patient acuity level (PAL) of a the main factor patient?

$21 / 28(75 \%)$ used professional judgement as the main factor $5 / 28(18 \%)$ used a mixture of professional judgement and the guidance as the main factor.

Confidence when assigning a PAL to a patient.

Mean confidence (SD) $=81 \%(20 \%)$

Median confidence $=82 \%$ (range $0 \%$ $100 \%)$

The PAST guidance helps me decide an appropriate PAL for each patient on the ward.

$4 / 28(14 \%)$ strongly agree

$20 / 28(71 \%)$ agree

$2 / 28(7 \%)$ neither agree or disagree

$2 / 28(7 \%)$ disagree

$0 / 28(0 \%)$ strongly disagree

My professional judgement is what mainly guides me when allocating a PAL for patient on the ward.

$14 / 28(50 \%)$ strongly agree

$12 / 28(43 \%)$ agree

$2 / 28(7 \%)$ neither agree or disagree

$0 / 28(0 \%)$ disagree

$0 / 28(0 \%)$ strongly disagree

When assessing a PAL, I use a mixture of my own professional judgement and the guideline.

$16 / 28(57 \%)$ strongly agree

9/28 (32\%) agree

$0 / 28(0 \%)$ neither agree or disagree

$1 / 28(4 \%)$ disagree

$2 / 28(7 \%)$ strongly disagree

Mean confidence (SD) $=70 \%(25 \%)$

Median confidence $=79 \%$ (range

$0 \%-100 \%)$

$3 / 28(11 \%)$ strongly agree

$6 / 28(21 \%)$ agree

$12 / 28(43 \%)$ neither agree or disagree

$6 / 28(21 \%)$ disagree

$1 / 28(4 \%)$ strongly disagree

PAST, pharmaceutical assessment screening tool.

in three of the case studies, 9/28 (32\%) pharmacists allocated a lower PAL, the research team found the same when they assessed the allocation of PALs in daily practice. ${ }^{7}$ The departmental pharmaceutical care standards state that a L3 patient should be seen every weekday by a senior pharmacist, a L2 patient should be seen by any pharmacist two or three times a week, and a L1 patient can be managed by a pharmacist or pharmacy technician unless medication or clinical condition of the patient changes.

All case study patients had different comorbidities and in three of the case studies the patients had one decompensated organ and were not on any high-risk medications, so would have been expected to have been classified as L2 using PAST. The different levels allocated for patients may again reflect the professional judgement of the pharmacists and experience on what they feel comfortable managing.

Experienced pharmacists may be allocating a lower PAL to a patient as they feel comfortable managing the patient at a lower level. In comparison, less experienced pharmacists may professionally feel less confident and want to assign a higher PAL to ensure the patient is seen more frequently and by a more experienced pharmacist.

The most notable variation in PAL choice was between the two L3 cases, 26/28 pharmacists (93\%) correctly allocated case study 4. The patient had one decompensated organ (the brain), active treatment for prostate cancer (goserelin) and he was also 'nil by mouth'. However, in case study 6, 21/28 pharmacists (75\%) assigned a lower level (L2) than PAST would have recommended (L3), this patient had two decompensated organs (the 
Table 2 Pharmacists' patient acuity level allocation to the case studies

\begin{tabular}{lcccccc}
\hline & Case study $\mathbf{1}(\mathrm{L} 2)$ & Case study $\mathbf{2}$ (L1) & Case study $\mathbf{3}$ (L2) & Case study 4 (L3) & Case study $\mathbf{5}$ (L2) & Case study 6 (L3) \\
\hline L1 & $0 / 28(0 \%)$ & $4 / 28(14 \%)$ & $0 / 28(0 \%)$ & $0 / 28(0 \%)$ & $4 / 28(14 \%)$ & $0 / 28(0 \%)$ \\
L2 & $22 / 28(79 \%)$ & $24 / 28(86 \%)$ & $20 / 28(71 \%)$ & $2 / 28(7 \%)$ & $22 / 28(79 \%)$ & $21 / 28(75 \%)$ \\
L3 & $6 / 28(21 \%)$ & $0 / 28(0 \%)$ & $8 / 28(29 \%)$ & $26 / 28(93 \%)$ & $2 / 28(7 \%)$ & $7 / 28(25 \%)$ \\
\hline
\end{tabular}

The bold text highlights the number of pharmacists that chose the correct PAL for the case study patient.

L1, level 1; L2, level 2; L3, level 3.

lung and kidneys). The difference may be due to the perceived severity of the decompensated organs and what pharmacists feel more competent to manage due to experience (eg, community acquired pneumonia and acute kidney injury are pharmaceutically managed more commonly than a subdural bleed and active prostate cancer).

In the other major deviation, 24/28 pharmacists (86\%) assigned case study 2 a higher PAL (L2) than recommended in PAST (L1). This patient had been diagnosed with a urinary tract infection and had been prescribed trimethoprim. The medical history of the patient included chronic back pain, angina, epilepsy and dementia, but the medication regimen was stable and included sodium valproate and regular codeine. Codeine is excluded from the high-risk medicines category as it is only a weak opiate but it is possible that the majority of pharmacists identified sodium valproate as a drug requiring therapeutic drug monitoring (even though serum levels are only used to detect non-adherence) thus allocating the patient the L2 status.

Overall the strength of agreement was 'fair', indicating that there was not a true agreement with PAST. ${ }^{8}$ This may reflect the overestimation and underestimation of allocated PALs seen in the case studies and again emphasises the pharmacist's use of professional clinical judgement when assigning a PAL. Exploration of the agreement between PAL extremes for the different levels of pharmacy experience showed that the strength of agreement was 'poor'. This suggests that the majority did not agree with the structured PAL guidance allocation for L2 patients. The overall results from this and our previous study ${ }^{7}$ indicate that the criteria for all the levels of the patients are clearly in need of further review, which should encourage the tool to be used more consistently in practise.

Confidence in an assessment screening tool may also be improved by ensuring the tool is fully validated to detect adverse drug events such as that designed by Urbina and colleagues in a Spanish hospital pharmacy department. ${ }^{9}$

The open text answers provided further insightful views about the limitations of the PAST. The most prominent limitation is that the PAST is at times used as a system to remind pharmacy team members that they need to follow-up a patient, especially on wards where there is not a regular pharmacy

Table 3 Results of the $\kappa$ statistic for pharmacists qualified a different number of years when allocating patient acuity level (PAL) extremes

\begin{tabular}{ll}
\hline & к Statistic $^{8}$ \\
\hline Qualified for $<1$ year expected PAL & 0.074 (poor) \\
Qualified for 1 to $<4$ years expected PAL & 0.081 (poor) \\
Qualified for 5 to $<10$ years expected PAL & 0.142 (poor) \\
Qualified for $>10$ years expected PAL & 0.073 (poor) \\
\hline PAL, patient acuity level. &
\end{tabular}

technician and the review may only involve a supply of medication. There was also a lack of confidence about how up-to-date the PAL was on the ward's patient identification boards after admission. This lack of confidence could mean that patients who do not need to be reviewed regularly are being reviewed, while patients who have become acutely unwell during their hospital admission are being overlooked.

Despite confident about using PAST, one-third of the respondents believe more training is needed to use it more effectively. Cottrell et al found that the usefulness of a pharmacy risk screening tool required substantial involvement by the pharmacy staff $^{3}$ and Hickson et $a l^{7}$ suggested that reiterating the true purpose of the tool could improve its reliability.

The strength of the study was that the questionnaire had an excellent response rate, which instils confidence that the results reflected the whole department and not solely either junior or senior pharmacists. A limitation is that the questionnaire results only reflect the attitudes of pharmacists at one hospital and so its application elsewhere may not be valid. Deeper insights into the use and understanding of the tool by pharmacists may also have been possible using more searching qualitative research methods, such as focus groups or interviews.

For future research, it would be beneficial to conduct intensive staff training after redesigning and validating the tool and then reassess adherence to it.

\section{What this paper adds}

What is already known on this subject?

- Pharmaceutical assessment screening tools are being developed and improved to help predict patient requirements for pharmaceutical care in hospitals and potentially reduce adverse drug events.

- Pharmacists may be using the tools to prioritise their own work schedule rather the pharmaceutical care needs of a clinical team or an entire hospital.

What this study adds?

- Pharmacists feel confident about using a pharmaceutical assessment screening tool to help them assign a patient acuity level and monitor pharmaceutical needs of patients while in hospital. However, the use of professional judgement to assign an acuity level overrides any predicted level from the tool itself.

- Careful design of validated screening tools, with appropriate training on their use, is required if such tools are to be successfully used by pharmacy departments to target which patients need to be seen more frequently, and by an appropriately experienced clinical pharmacist, and ultimately fulfil their promise to prevent adverse drug events in hospital inpatients. 


\section{CONCLUSION}

Pharmacists feel confident about using PAST to help them assign a PAL and monitor pharmaceutical needs of patients while in hospital. However, the use of professional judgement to assign a PAL overrides any predicted level from the tool itself.

Careful design of validated screening tools, with appropriate training on their use, is required if such tools are to be successfully used by pharmacy departments to target which patients need to be seen more frequently, and by an appropriately experienced clinical pharmacist, and ultimately fulfil their promise to prevent adverse drug events in hospital inpatients.

Contributors This research contributes to further understanding the importance of the opinions and attitudes of the pharmacists using a prioritisation tool in practice and how to ensure confidence when using the tool.

Competing interests SDW is an associate editor for the European Journal of Hospital Pharmacy.

Provenance and peer review Not commissioned; externally peer reviewed.

\section{REFERENCES}

1 Intensive Care Society. Levels of Critical Care for Adult Patients. Intensive Care Society Standards (revised 2009). London: The Intensive Care Society, 2009. http:/ www.ics.ac.uk/ics-homepage/guidelines-and-standards/ (accessed May 2016).
2 The Safer Nursing Care Tool (SNCT). The Shelford Group. 2014. http:/l shelfordgroup.org/library/documents/SNCT_A4_23May14a.pdf (accessed May 2016).

3 Cottrell R, Caldwell M, Jardine G. Developing and implementing a pharmacy riskscreening tool. Hospital Pharmacy Europe, 2013. http://www. hospitalpharmacyeurope.com/featured-articles/developing-andimplementing-pharmacy-risk-screening-tool (accessed May 2016).

4 Falconer N, Nand S, Liow D, et al. Development of an electronic patient prioritization tool for clinical pharmacist interventions. Am J Health Syst Pharm 2014; 71:311-20.

5 Mullan N, Jennings A. Pharmacists' Use and Views of the Electronic Prescribing Web Portal [abstract]. GHP/UKCPA Joint National Conference 2013. http://www.ukcpa.net/ wp-content/uploads/2014/06/Joint-Conference-2014-Abstracts-only.pdf (accessed May 2016).

6 Safadeh M, Pazik L, Kavangh R. A baseline assessment of the pharmaceutical needs of adult patients admitted to Stoke Mandeville Hospital [abstract]. Clin Pharm 2012: (Suppl 2):S36-8. http://www.ukcpa.net/wp-content/uploads/2012/06/ Autumn-Symposium-November-2011.pdf

7 Hickson RP, Steinke DT, Skitterall C, et al. Evaluation of a pharmaceutical assessment screening tool to measure patient acuity and prioritise pharmaceutical care in a UK hospital. Eur J Hosp Pharm 2016:10:1136.

8 Dawson B, Trapp RG. Basic and clinical biostatistics. London: Lang Medical Books/ McGraw-Hill. 2004:118.

9 Urbina, Ferrández 0 , Luque $S$, et al. Patient risk factors for developing a drug-related problem in a cardiology ward. Ther Clin Risk Manag 2015: 11:9-15. 